\title{
Discrete to Scale-Dependent Continua for Complex Materials: A Generalized Voigt Approach Using the Virtual Power Equivalence
}

Patrizia Trovalusci

\begin{abstract}
The mechanical behaviour of complex materials, characterised at finer scales by the presence of heterogeneities of significant size and texture, strongly depends on their microstructural features. By lacking in material internal scale parameters, the classical continuum does not always seem appropriate for describing the macroscopic behaviour of such materials, taking into account the size, the orientation and the disposition of the heterogeneities. This often calls for the need of non-classical continuum descriptions, which can be obtained through multiscale approaches aimed at deducing properties and relations by bridging information at different levels of material descriptions.

Current researches in solid state physics as well as in mechanics of materials show that energy-equivalent continua obtained by defining direct links with lattice systems, as widely investigated by the corpuscular-continuous approaches of $19^{\text {th }}$ century, are still among the most promising approaches in material science. The aim is here to point out the suitability of adopting discrete to scale-dependent continuous models, based on a generalization of the so-called Cauchy-Born (Voigt) rule used in crystal elasticity and in classical molecular theory of elasticity, in order to identify continua with additional degrees of freedom (micromorphic, multifield, etc.) which are essentially non-local models with internal length and dispersive properties. It is shown that, within the general framework of the principle of virtual powers, the correspondence map relating the finite number of degrees of freedom of discrete models to the continuum kinematical fields provides a guidance on the choice of the most appropriate continuum approximation for heterogeneous media. Some applications of the mentioned approach to ceramic matrix composites and masonry-like materials are discussed.
\end{abstract}

Patrizia Trovalusci

Department of Structural and Geotechnical Engineering, Sapienza University of Rome, Italy, email: patrizia.trovalusci@uniroma1.it 


\section{Introduction}

This contribution focuses on constitutive theories for continuous models originated from refined discontinuous descriptions of materials. The classical molecular theory of elasticity, as developed by Navier, Cauchy and Poisson [25, 7, 30] in the $19^{\text {th }}$ century, represents the first attempt to derive the field equations of an elastic body basing on the definition of microscopic laws for systems of point-like particles ('molecules') close together and interacting through attractive forces. In these mechanistic descriptions, inspired to Newton's idea of coherence of elastic bodies [26], the molecules, are perceived as ultimate particles without extension, inside which no forces are accounted for, that interact in pairs through forces depending on their mutual distance and directed along the line connecting their centres ('central-force' scheme). A kinematic corresponding map between the discrete degrees of freedom and the continuous fields guarantees the transition from the fine to the gross description. Macroscopic stress measures are then derived as averages of molecular material quantities over a convenient volume element, called 'molecular sphere of action', outside which intermolecular forces are negligible.

The central-force description led to experimental discrepancies concerning the number of elastic constants, that were less than those needed to represent the behaviour of materials belonging to various symmetry classes. Successively, Voigt and Poincaré introduced mixed energetic/mechanistic approaches providing refined molecular models that circumvented the problem of the underestimation of the number of the material constants related to the central-force scheme [32, 2, 3, 31]. In particular, Voigt introduced a potential of force and moment interactions exerted between pairs of rigid bodies, while Poincaré proposed a multibody potential description [35, 36, 29]. Both Voigt and Poincaré removed the local character of the Cauchy description by modifying the central-force scheme thus obtaining continua which could be classifiable as 'implicitly' or 'weakly' non-local [18, 20, 10], because of the presence of internal lengths and dispersion properties that can be there recognized [31]; although they finally led back to classical continua by introducing internal constraints: Voigt by imposing the same uniform rotation to the particles and Poincaré by considering only pair-interaction terms. However, even if both Voigt and Poincaré, on using the refinement of non-local descriptions, offered a good solution to the controversy about the elastic constants, the mechanistic-molecular approach was abandoned in favour of the energetic-continuum approach by Green, and their works have been neglected for long time [3].

Now these ideas found a renewed interest with particular reference to the problem of constitutive modelling of composite materials. The mechanical behaviour of materials characterized at finer scales by the presence of heterogeneities of significant size and texture strongly depends on their internal structure that is intrinsically heterogeneous and discrete because interfaces (grain boundaries, thin layers, etc.) dominate the gross behaviour. By lacking in material internal scale parameters, moreover, the classical continuum does not always seem appropriate to describe the macroscopic behaviour of such materials taking into account the size, the orientation and the disposition of the heterogeneities. This calls for the need of non-local con- 
tinuum descriptions which can still be obtained through homogenization approaches aimed at deducing properties and relations by bridging information at proper underlying discrete micro-levels via energy equivalence criteria. Thus, providing that the original lattice models were refined by extending the concept of molecule in order to describe different internal phases (rigid inclusions, voids, etc.) and taking into account of non central inter-molecular actions, or by enriching the potential descriptions introducing multibody interaction terms, discrete-to-scale dependent non-local continua could be naturally derived. As sample models nonlocal continua with additional degrees of freedom are reported. These continua are non-classical continua, of the kind described in $[22,5,10,14,1]$, derived from lattice systems made of rigid particles and distributed voids (pores, microcracks, etc.) and adopted for the description of ceramic matrix composites or masonry-like materials.

\section{Corpuscular micro-model}

The discrete model adopted for the fine description of the reference composite material is made of kind of structured molecules broadly representing the internal phases of the material: the fibres, described as rigid particles of polygonal shape, and the flaws, perceived as slits of arbitrary shape and a predominant dimension. The slits are considered opened, stationary and with blunt edges (no tip effects accounted for). The particles interact in pairs through forces and couples while the slits interact through forces directed along the line connecting their centres. Particles and slits also interact each other by forces. The slits must be considered as devices to transmit to the matrix additional forces due to the presence of defects. In this sense they represent the microcracks/pores. Their stiffness depends on the surrounding elastic field. In this paragraph the analysis is conducted within the linearised framework, where the velocity fields stand for infinitesimal displacement fields and the power stands for work.

Let $\mathscr{A}$ and $\mathscr{B}$ be two rigid particles, respectively centred at the positions a and $\mathbf{b}$, and $\mathscr{H}$ and $\mathscr{K}$ two slits located at the positions $\mathbf{h}$ and $\mathbf{k}$. The vectors $\mathbf{w}^{a}$ and $\mathbf{w}^{b}$ respectively denote the velocity of $\mathbf{a}$ and $\mathbf{b}$ and the skew-symmetric tensors $\mathbf{W}^{a}$ and $\mathbf{W}^{b}$ the angular velocities of the two particles. For each pair of adjacent particles the strain measures of the lattice are defined as:

$$
\begin{aligned}
\mathbf{w}_{i} & =\mathbf{w}_{i}^{a}-\mathbf{w}_{i}^{b}=\left[\mathbf{w}^{a}+\mathbf{W}^{a}\left(\mathbf{p}^{a}-\mathbf{a}\right)\right]-\left[\mathbf{w}^{b}+\mathbf{W}^{b}\left(\mathbf{p}^{b}-\mathbf{b}\right)\right], \\
\mathbf{W}_{i} & =\mathbf{W}^{a}-\mathbf{W}^{b},
\end{aligned}
$$

where $\mathbf{p}^{a}$ and $\mathbf{p}^{b}$ are two test points, on $\mathscr{A}$ and $\mathscr{B}$, through which the particles interact, and $\mathbf{w}_{i}^{a}$ and $\mathbf{w}_{i}^{b}$ their velocities. Further lattice strain measures for each slit $\mathscr{H}$, each pair of interacting slits $(\mathscr{H}, \mathscr{K})$ and each pair of interacting particle-slit $(\mathscr{A}, \mathscr{H})$ are:

$$
\mathbf{d}^{h}, \quad \mathbf{d}_{j}=\mathbf{d}^{h}-\mathbf{d}^{k}, \quad \mathbf{w}_{l}=\mathbf{w}_{i}^{a}-\left(\mathbf{w}^{h}+\mathbf{d}^{h}\right),
$$


where: the vector $\mathbf{d}^{h}\left(\mathbf{d}^{k}\right)$ represents the half-crack opening displacement on $\mathscr{H}$ $(\mathscr{K})$ and $\mathbf{w}^{h}\left(\mathbf{w}^{k}\right)$ is the velocity vector of $\mathbf{h}(\mathbf{k})$ on the external boundary of $\mathscr{H}$ $(\mathscr{K})$.

The forces and the couples that $\mathscr{B}(\mathscr{A})$ exerts on $\mathscr{A}(\mathscr{B})$ are respectively represented by the vector $\mathbf{t}^{a}\left(\mathbf{t}^{b}\right)$ and the skew-symmetric tensor $\mathbf{C}^{a}\left(\mathbf{C}^{b}\right)$. The force due to $\mathbf{d}^{h}$ on $\mathscr{H}$ is represented by the vector $\mathbf{z}_{o}^{h}$. Due to $\mathbf{d}^{h}$, the slit interacts with the adjacent particles and the neighbouring slits. The vector $\mathbf{z}^{h}\left(\mathbf{z}^{k}\right)$ is the action that $\mathscr{K}$ $(\mathscr{H})$ exerts on $\mathscr{H}(\mathscr{K})$, while the vector $\mathbf{r}^{h}\left(\mathbf{r}^{a}\right)$ represents the action transmitted by $\mathscr{H}(\mathscr{A})$ to $\mathscr{A}(\mathscr{H})$.

If the material can be considered periodic, or at least statistically homogeneous, a representative volume element, $\mathscr{M}_{\mu}$, referred as the module, can be individuated. Taking into account the balance equations of the internal actions of the module the mean power of the internal actions over the volume of the module, $V\left(\mathscr{M}_{\mu}\right)$, can be written:

$$
\begin{aligned}
\bar{\Pi}_{\mu}= & \frac{1}{V\left(\mathscr{M}_{\mu}\right)}\left\{\sum_{i}\left\{\mathbf{t}_{i} \cdot\left[\mathbf{w}_{i}-\mathbf{W}^{a}\left(\mathbf{p}^{a}-\mathbf{p}^{b}\right)\right]+\frac{1}{2} \mathbf{C}_{i} \cdot \mathbf{W}_{i}\right\}\right. \\
& \left.+\sum_{h} \mathbf{z}_{o}^{h} \cdot \mathbf{d}^{h}+\sum_{j} \mathbf{z}_{j} \cdot \mathbf{d}_{j}+\sum_{l}\left\{\mathbf{r}_{l} \cdot\left[\mathbf{w}_{l}-\mathbf{W}^{a}\left(\mathbf{p}^{a}-\mathbf{h}\right)\right]\right\}\right\},
\end{aligned}
$$

where it has been put: $\mathbf{t}^{a}=-\mathbf{t}^{b}=\mathbf{t}_{i}, \mathbf{z}^{h}=-\mathbf{z}^{k}=\mathbf{z}_{j}, \mathbf{r}^{h}=-\mathbf{r}^{a}=\mathbf{r}_{l}$,

$\mathbf{C}^{a}=-\mathbf{C}^{b}+\left[\left(\mathbf{p}^{a}-\mathbf{p}^{b}\right) \otimes \mathbf{t}^{a}-\mathbf{t}^{a} \otimes\left(\mathbf{p}^{a}-\mathbf{p}^{b}\right)\right]+\left[\left(\mathbf{p}^{a}-\mathbf{h}\right) \otimes \mathbf{r}^{h}-\mathbf{r}^{h} \otimes\left(\mathbf{p}^{a}-\mathbf{h}\right)\right]=\mathbf{C}_{i}$,

and where the summations are respectively extended to each pair $(\mathscr{A}, \mathscr{B}),(\mathscr{H}, \mathscr{K})$, $(\mathscr{A}, \mathscr{H})$ in $\mathscr{M}_{\mu}$.

The selection of linear elastic response functions for the interactions between particles and for the forces due to the crack opening displacements; non-linear elastic functions for the interactions between slits, decribed as continuous distributions of dislocations with Burgers' vector parallel to the opening directions; other nonlinear phenomenological functions for the interactions between slits and particles:

$$
\begin{array}{rlrl}
\mathbf{t}_{i} & =\mathbf{K}_{i}\left[\mathbf{w}_{i}-\mathbf{W}^{a}\left(\mathbf{p}^{a}-\mathbf{p}^{b}\right)\right], & \mathbf{C}_{i} & =\mathrm{K}_{i} \mathbf{W}_{i}, \quad \mathbf{z}_{o}^{h}=\mathbf{D}^{h} \mathbf{d}^{h}, \\
\mathbf{z}_{j} & =D_{j} \frac{\left\|\mathbf{d}^{h}\right\|\left\|\mathbf{d}^{k}\right\|}{\|\mathbf{h}-\mathbf{k}\|^{2}}(\mathbf{h}-\mathbf{k}), & \mathbf{r}_{l}=\frac{f_{1}(\mathbf{a}) f_{2}(\mathbf{h})}{\|\mathbf{a}-\mathbf{h}\|^{2}}(\mathbf{a}-\mathbf{h}),
\end{array}
$$

here assumes a purely paradigmatic meaning for the procedure reported in Section 3 and it can at any time be modified in order to meet the needs of finer constitutive descriptions. In Equations (4) the components of the second order $\mathbf{K}_{i}, \mathbf{D}^{h}$ and fourth order $\mathrm{K}_{i}$ tensors; the constant $D_{j}$ and the scalar functions $f_{1}$ and $f_{2}$ (two approximately Gaussian functions describing respectively the local force field around a particle $(\mathscr{A})$ and a slit ( $\mathscr{H})$ [19]) depend on the elastic constants of the matrix and the geometry of the two kinds of inclusions. 


\section{Micro-macro transition via virtual power equivalence}

In order to identify the equivalent continuum model, hypotheses of regularity of the kinematical descriptors introduced in Section 2 are given. According to discretecontinuum coarse-graining approaches described in [31], kinematical maps relating discrete-to-continuous kinematical fields are introduced. These maps are given by Taylor expansions up to the second order of the macro velocity vector $\mathbf{w}(\mathbf{x})$, the skew-symmetric micro angular velocity tensor $\mathbf{W}(\mathbf{x})$, and the independent micro velocity vector $\mathbf{d}(\mathbf{x})$ :

$$
\begin{aligned}
\mathbf{w}^{a} & =\mathbf{w}(\mathbf{x})+\nabla \mathbf{w}(\mathbf{x})(\mathbf{a}-\mathbf{x})+\frac{1}{2}\left[\nabla^{2} \mathbf{w}(\mathbf{x})(\mathbf{a}-\mathbf{x})\right](\mathbf{a}-\mathbf{x})+o(\mathbf{a}-\mathbf{x}) \\
\mathbf{W}^{a} & =\mathbf{W}(\mathbf{x})+\nabla \mathbf{W}(\mathbf{x})(\mathbf{a}-\mathbf{x})+\frac{1}{2}\left[\nabla^{2} \mathbf{W}(\mathbf{x})(\mathbf{a}-\mathbf{x})\right](\mathbf{a}-\mathbf{x})+o(\mathbf{a}-\mathbf{x}) \\
\mathbf{d}^{h} & =\mathbf{d}(\mathbf{x})+\nabla \mathbf{d}(\mathbf{x})(\mathbf{h}-\mathbf{x})+\frac{1}{2}\left[\nabla^{2} \mathbf{d}(\mathbf{x})(\mathbf{h}-\mathbf{x})\right](\mathbf{a}-\mathbf{x})+o(\mathbf{h}-\mathbf{x}),
\end{aligned}
$$

for any $\mathscr{A}, \mathscr{H} \in \mathscr{M}_{\mu}$, where $\mathbf{x}$ is the centre of the module and where, from now on, the term 'macro' stands for standard and 'micro' for non-standard fields. Assuming that a continuous neighborhood $\mathscr{M}$ of $\mathbf{x}$, occupying the same Euclidean region of $\mathscr{M}_{\mu}$, is well definede, these maps impose that the continuum locally undergoes the same deformations as the lattice system. Equations (5) provide a generalization of Cauchy, Voigt or Poincaré's homogenization rules reported in [31]. From now on, the explicit dependence of any field on $\mathbf{x}$ will be undertaken.

Basing on the maps (5) various kinds of continua can be identified that are in general non-classical, as described in [31]. By expanding the series up to higher orders refined descriptions allowing to take into account long-range interactions can be obtained. Specific continuous models can also be derived by imposing proper internal constraints to the lattice model, as in the cases studied by Voigt and Poincaré, obtaining continua that can be defined continua with latent microstructure [4].

\subsection{First order continuum approximation. Continuum with rigid and affine local structure}

Using Equations (5) with $\nabla \mathbf{w}, \nabla \mathbf{W}$ and $\nabla \mathbf{d}$ constant the strain measures of the lattice system (1), (2) can be expressed in terms of the smooth fields $\nabla \mathbf{w}-\mathbf{W}, \nabla \mathbf{W}, \mathbf{d}$ and $\nabla \mathbf{d}$ as:

$$
\begin{aligned}
\mathbf{w}_{i} & =(\nabla \mathbf{w}-\mathbf{W})(\mathbf{a}-\mathbf{b})+\nabla \mathbf{W}\left[\left(\mathbf{p}^{a}-\mathbf{a}\right) \otimes(\mathbf{a}-\mathbf{x})-\left(\mathbf{p}^{b}-\mathbf{b}\right) \otimes(\mathbf{a}-\mathbf{x})\right] \\
\mathbf{W}_{i} & =\nabla \mathbf{W}(\mathbf{a}-\mathbf{b}) \\
\mathbf{d}_{j} & =\nabla \mathbf{d}(\mathbf{h}-\mathbf{k})
\end{aligned}
$$

where the explicit dependence of any field on $\mathbf{x}$ has been undertaken. 
After some algebra, the mean power of the contact actions (3) can be also expressed as function of these strain fields:

$$
\begin{array}{rlrl}
\bar{\Pi}_{\mu} & =\frac{1}{V(\mathscr{M} \mu)}\left\{\left\{\sum_{i} \mathbf{t}_{i} \otimes(\mathbf{a}-\mathbf{b})+\sum_{i} \mathbf{r}_{l} \otimes(\mathbf{a}-\mathbf{h})\right\} \cdot(\nabla \mathbf{w}-\mathbf{W})\right. \\
& + & & \left\{\sum_{i} \mathbf{t}_{i} \otimes\left[\left(\mathbf{p}^{a}-\mathbf{a}\right) \otimes(\mathbf{a}-\mathbf{x})-\left(\mathbf{p}^{b}-\mathbf{b}\right) \otimes(\mathbf{b}-\mathbf{x})\right]+\frac{1}{2} \mathbf{C}_{i} \otimes(\mathbf{a}-\mathbf{b})\right\} \cdot \nabla \mathbf{W} \\
& + & \left\{\sum_{h} \mathbf{z}_{o}^{h}+\sum_{l} \mathbf{r}_{l}\right\} \cdot \mathbf{d} \\
+ & \left.\left\{\sum_{h} \mathbf{z}_{o}^{h} \otimes(\mathbf{h}-\mathbf{x})+\sum_{j} \mathbf{z}_{j} \otimes(\mathbf{h}-\mathbf{k})+\sum_{l} \mathbf{r}_{l} \otimes(\mathbf{h}-\mathbf{x})\right\} \cdot \nabla \mathbf{d}\right\}
\end{array}
$$

It can be now assumed that a continuum scalar field representing the internal power density of a multifield continuum, in a neighbourhood of $\mathbf{x}$ occupying the same region of the module $\mathscr{M} \equiv \mathscr{M}_{\mu}$, exists as a function of the primal strain fields $\nabla \mathbf{w}-\mathbf{W}, \nabla \mathbf{W}, \mathbf{d}, \nabla \mathbf{d}$ :

$$
\pi(\nabla \mathbf{w}-\mathbf{W}, \mathbf{d}, \nabla \mathbf{d})=\mathbf{S} \cdot(\nabla \mathbf{w}-\mathbf{W})+\frac{1}{2} S \cdot \nabla \mathbf{W}+\mathbf{z} \cdot \mathbf{d}+\mathbf{Z} \cdot \nabla \mathbf{d},
$$

where the second order tensor $\mathbf{S}$, the third order tensor $\mathbf{S}$, the vector $\mathbf{z}$ and the second order tensor $\mathbf{Z}$ are the dual stress fields power-conjugate to the strain measures $\nabla \mathbf{w}-\mathbf{W}, \nabla \mathbf{W}, \mathbf{d}, \nabla \mathbf{d}$, respectively.

The requirement that the internal power is preserved in the transition from the fine to the gross description for any $\nabla \mathbf{w}-\mathbf{W}, \nabla \mathbf{W}, \mathbf{d}$ and $\nabla \mathbf{d}$, through the localization theorem, gives:

$$
\bar{\Pi}_{\mu}(\nabla \mathbf{w}-\mathbf{W}, \mathbf{d}, \nabla \mathbf{d})=\pi(\nabla \mathbf{w}-\mathbf{W}, \mathbf{d}, \nabla \mathbf{d}) .
$$

Then the continuum stress measures are identified as functions of the internal actions and of the fabric vector and tensors of the module (i.e. size, shape and disposition of inclusions):

$$
\begin{aligned}
& \mathbf{S}=\frac{1}{V\left(\mathscr{M}_{\mu}\right)}\left\{\sum_{i} \mathbf{t}_{i} \otimes(\mathbf{a}-\mathbf{b})+\sum_{l} \mathbf{r}_{l} \otimes(\mathbf{a}-\mathbf{h})\right\}, \\
& \mathrm{S}=\frac{1}{V\left(\mathscr{M}_{\mu}\right)}\left\{\sum_{i} 2 \mathbf{t}_{i} \otimes\left[\left(\mathbf{p}^{a}-\mathbf{a}\right) \otimes(\mathbf{a}-\mathbf{x})-\left(\mathbf{p}^{b}-\mathbf{b}\right) \otimes(\mathbf{b}-\mathbf{x})\right]+\mathbf{C}_{i} \otimes(\mathbf{a}-\mathbf{b})\right\}, \\
& \mathbf{z}=\frac{1}{V(\mathscr{M} \mu)}\left\{\sum_{h} \mathbf{z}_{o}^{h}+\sum_{l} \mathbf{r}_{l}\right\} \\
& \mathbf{Z}=\frac{1}{V\left(\mathscr{M}_{\mu}\right)}\left\{\sum_{h} \mathbf{z}_{o}^{h} \otimes(\mathbf{h}-\mathbf{x})+\sum_{j} \mathbf{z}_{j} \otimes(\mathbf{h}-\mathbf{k})+\sum_{l} \mathbf{r}_{l} \otimes(\mathbf{h}-\mathbf{x})\right\} .
\end{aligned}
$$

In the virtual power setting delineated, non variational, the results apply regardless of the material response. Once the constitutive equations for the lattice system are defined, for instance those of Equations (4), by identifying the actual strain rates of the discrete model using again the maps (5), always under the hypothesis of homogeneous deformations, the continuum constitutive relations for all the stress 
measures introduced are derived in the following form:

$$
\begin{aligned}
& \mathbf{S}=\mathrm{A}(\nabla \mathbf{w}-\mathbf{W})+\mathrm{B} \nabla \mathbf{W}+\mathrm{Cd}+\mathrm{D} \nabla \mathbf{d}+\Psi_{\mathrm{S}}\left(\mathbf{d}^{2}, \nabla \mathbf{d}^{2},\|\mathbf{d}\|\|\nabla \mathbf{d}\|\right), \\
& \mathrm{S}=\mathrm{E}(\nabla \mathbf{w}-\mathbf{W})+\mathrm{F} \nabla \mathbf{W}, \\
& \mathbf{z}=\mathrm{I}(\nabla \mathbf{w}-\mathbf{W})+\mathbf{M d}+\mathrm{N} \nabla \mathbf{d}+\Psi_{\mathbf{Z}}\left(\mathbf{d}^{2}, \nabla \mathbf{d}^{2},\|\mathbf{d}\|\|\nabla \mathbf{d}\|\right), \\
& \mathbf{Z}=\mathrm{O}(\nabla \mathbf{w}-\mathbf{W})+\mathrm{Q} \mathbf{d}+\mathrm{R} \nabla \mathbf{d}+\Psi_{\mathbf{Z}}\left(\mathbf{d}^{2}, \nabla \mathbf{d}^{2},\|\mathbf{d}\|\|\nabla \mathbf{d}\|\right) .
\end{aligned}
$$

In Equations (11) the constitutive tensors of the second (M), third (C, I, N, Q), fourth $(A, D, O, R)$, fifth $(B, E)$ and sixth $(F)$ order have components depending on the elastic constants and the geometrical parameters of the material phases, as well as the non-linear vector $\left(\Psi_{\mathbf{Z}}\right)$ and second order tensor $\left(\Psi_{\mathbf{S}}, \Psi_{\mathbf{Z}}\right)$ functions. If the discrete system is hyperelastic, also the equivalent continuum is hyperelastic and the following symmetry relations between constitutive tensors hold: BT $\cdot \mathbf{T}=\mathrm{T} \cdot \mathrm{ET}$, for any third order tensor $T$ and second order tensor $\mathbf{T} ; \mathbf{C} \mathbf{v} \cdot \mathbf{T}=\mathbf{v} \cdot \mid \mathbf{T}$, for any vector $\mathbf{v}$ and second order tensor $\mathbf{T}$; DT $\cdot \mathbf{V}=\mathbf{T} \cdot \mathrm{OV}$, for any second order tensor $\mathbf{T}$ and $\mathbf{V} ; \mathbf{N T} \cdot \mathbf{v}=\mathbf{T} \cdot \mathbf{Q v}$, for any second order tensor $\mathbf{T}$ and vector $\mathbf{v}$. If the material microstructure is arranged respecting the central symmetry the odd order tensors B, C, $\mathrm{N}$, and the corresponding transposed tensors defined by the above relations, are null. Moreover, the tensors B, C, F, M, N, as well as the corresponding transposed tensors, contain internal length parameters and then, even in this case of homogeneous deformations, the non-local character of the description is guaranteed.

The lattice system described in Section 2 can be then replaced by an equivalent multifield continuum with additional degrees of freedom endowed with a rigid local structure (Cosserat, e.g. [10]) plus a deformable (affine) structure, of the kind described in [5] or also in [14], encoded in the power formula (8). This continuum undergo microdeformations independent of the local macroscopic deformation and a detailed description of the basics can be found in $[34,31]$. This description can be contextualized within the more general frameworks delineated in $[27,8,11,16]$.

In the case in which $\mathbf{d}=\mathbf{0}$, using Equations (11) where no-interaction between particle and slits are accounted for $(C=0, I=0$ and $D=0, O=0)$, the internal power density (8) can be written:

$$
\pi=[\mathrm{A}(\nabla \mathbf{w}-\mathbf{W})+\mathrm{B} \nabla \mathbf{W}] \cdot(\nabla \mathbf{w}-\mathbf{W})+\frac{1}{2}[\mathrm{E}(\nabla \mathbf{w}-\mathbf{W})+\mathrm{F} \nabla \mathbf{W}] \cdot \nabla \mathbf{W},
$$

that is the power density formula of a micropolar continuum equivalent to an assembly of rigid particles, without slits, undergoing independent rotations one each other, $\mathbf{W}^{a}$, and interacting through forces and couples, $\mathbf{t}^{a}, \mathbf{C}^{a}\left(\forall \mathscr{A}\right.$ in $\left.\mathscr{M}_{\mu}\right)$.

In order to make comparisons with other continuous models, it is useful to distinguish in the expression (12) the contributions of the symmetric and the skewsymmetric part of the strain and stress tensors. By decomposing the displacement gradient $\nabla \mathbf{w}=\mathbf{E}+\mathbf{R}$, with $\mathbf{E}=\operatorname{sym}[\nabla \mathbf{w}]$ and $\mathbf{R}=\operatorname{skw}[\nabla \mathbf{w}]$, where the operators sym and skw respectively extract the symmetric and the skew-symmetric part of a tensor, it is: 


$$
\begin{aligned}
\pi & =\operatorname{sym}[\mathrm{A}(\mathbf{E}+\mathbf{R}-\mathbf{W})+\mathrm{B} \nabla \mathbf{W}] \cdot \mathbf{E}+\operatorname{skw}[\mathrm{A}(\mathbf{E}+\mathbf{R}-\mathbf{W})+\mathrm{B} \nabla \mathbf{W}] \cdot(\mathbf{R}-\mathbf{W}) \\
& +\frac{1}{2}[\mathrm{E}(\mathbf{E}+\mathbf{R}-\mathbf{W})+\mathrm{F} \nabla \mathbf{W}] \cdot \nabla \mathbf{W} .
\end{aligned}
$$

By putting:

$$
\begin{aligned}
\pi^{Y Y} & =\operatorname{sym}[\mathrm{AE}] \cdot \mathbf{E} \\
\pi^{Y K} & =\operatorname{sym}[\mathrm{A}(\mathbf{R}-\mathbf{W})] \cdot \mathbf{E}=\operatorname{skw}[\mathrm{AE}] \cdot(\mathbf{R}-\mathbf{W})=\pi^{K Y} \\
\pi^{K K} & =\operatorname{skw}[\mathrm{A}(\mathbf{R}-\mathbf{W})] \cdot(\mathbf{R}-\mathbf{W}) \\
\pi^{Y C} & =\operatorname{sym}[\mathrm{B} \nabla \mathbf{W}] \cdot \mathbf{E}=\frac{1}{2} \mathrm{EE} \cdot \nabla \mathbf{W}=\pi^{C Y} \\
\pi^{K C} & =\operatorname{skw}[\mathrm{B} \nabla \mathbf{W}] \cdot(\mathbf{R}-\mathbf{W})=\frac{1}{2} \mathrm{E}(\mathbf{R}-\mathbf{W}) \cdot \nabla \mathbf{W}=\pi^{C K} \\
\pi^{C C} & =\frac{1}{2} \mathrm{~F} \nabla \mathbf{W} \cdot \nabla \mathbf{W},
\end{aligned}
$$

Equation (13) can be written:

$$
\pi=\pi^{Y Y}+\pi^{K K}+\pi^{C C}+2\left(\pi^{Y K}+\pi^{Y C}+\pi^{K C}\right) .
$$

It can be noticed that the term $\pi^{Y Y}$ corresponds to the classical term. The terms $\pi^{K K}$ and $\pi^{C C}$ are characteristic to the Cosserat continuum. The mixed terms $\pi^{C Y}=\pi^{Y C}$ and $\pi^{C K}=\pi^{K C}$ are null in the case of materials belonging to the class of centrosymmetric materials or more restricted symmetry classes, while the mixed term $\pi^{Y K}=\pi^{K Y}$ is null in the case of orthotetragonal materials or more restricted material symmetry classes.

\subsection{Second order continuum approximation}

Let us now consider the case in which, in the maps (5), $\mathbf{d}=\mathbf{0}$ and $\mathbf{W}=\operatorname{skw}[\nabla \mathbf{w}]=$ $\mathbf{R}$, with $\nabla^{2} \mathbf{w} \neq \mathbf{0}$. Referring to the original lattice system, these constraints connote a system without slits and with particles constrained to undergo the same local rigid rotation of the continuum $\left(\mathbf{W}^{a}=\mathbf{R}, \forall(\mathscr{A}) \in \mathscr{M}_{\mu}\right)$, as in the Voigt model described in [31]. This implies that: $\nabla \mathbf{w}-\mathbf{W}=\mathbf{E}(\mathbf{E}=\operatorname{sym}[\nabla \mathbf{w}])$ and, for negligible distances $\mathbf{p}^{a}-\mathbf{p}^{b}$, the strain measures of the lattice reduce to:

$$
\mathbf{w}_{i}=\mathbf{E}(\mathbf{a}-\mathbf{b})+\frac{1}{2} \nabla^{2} \mathbf{w}[(\mathbf{a}-\mathbf{x}) \otimes(\mathbf{a}-\mathbf{x})-(\mathbf{b}-\mathbf{x}) \otimes(\mathbf{b}-\mathbf{x})],
$$

where the explicit dependence of any field on $\mathbf{x}$ is undertaken. Hence, the mean power of the contact actions over $\mathscr{M}_{\mu}$ (3) can be written as: 


$$
\begin{aligned}
\bar{\Pi}_{\mu}= & \frac{1}{V\left(\mathscr{M}_{\mu}\right)}\left\{\sum_{i} \mathbf{t}_{i} \otimes(\mathbf{a}-\mathbf{b}) \cdot \mathbf{E}\right. \\
& \left.+\frac{1}{2} \sum_{i} \mathbf{t}_{i} \otimes[(\mathbf{a}-\mathbf{x}) \otimes(\mathbf{a}-\mathbf{x})-(\mathbf{b}-\mathbf{x}) \otimes(\mathbf{b}-\mathbf{x})] \cdot \nabla^{2} \mathbf{w}\right\} .
\end{aligned}
$$

This formula corresponds to the mean power of the internal action of the module of a lattice system whose particles, as mentined above, are locally constrained to have the same rotation and to (non-locally) interact through forces and moments of forces, but no couples. It can be shown in fact that, for $\mathbf{p}^{a}-\mathbf{p}^{b}$ approaching to zero, constitutive and balance considerations imply that the interaction couple $\mathbf{C}_{i}$ is null (as assumed by Voigt, [36], p. 599).

Considering a continuous neighborhood, $\mathscr{M} \equiv \mathscr{M}_{\mu}$, of a second-gradient continuum of the kind described in [23, 24], which has the fields $\mathbf{E}$ and $\nabla^{2} \mathbf{w}$ as primal strain fields, the equivalence between the mean internal power of the module (Equation 17) and the internal power density formula of the continuum

$$
\pi=\mathbf{T} \cdot \mathbf{E}+\mathrm{T} \cdot \nabla^{2} \mathbf{w},
$$

for any $\mathbf{E}$ and $\nabla^{2} \mathbf{w}$, through the localization theorem, gives the dual stress measures as functions of the contact actions and of the fabric quantities of the module:

$$
\begin{aligned}
& \mathbf{T}=\frac{1}{V\left(\mathscr{M}_{\mu}\right)} \sum_{i} \operatorname{sym}\left[\mathbf{t}_{i} \otimes(\mathbf{a}-\mathbf{b})\right] \\
& \mathrm{T}=\frac{1}{2 V\left(\mathscr{\mathscr { M }}_{\mu}\right)} \sum_{i} \mathbf{t}_{i} \otimes[(\mathbf{a}-\mathbf{x}) \otimes(\mathbf{a}-\mathbf{x})-(\mathbf{b}-\mathbf{x}) \otimes(\mathbf{b}-\mathbf{x})] .
\end{aligned}
$$

Assuming that the interactions between the pairs of particles $(\mathscr{A}, \mathscr{B})$ are linear elastic forces: $\mathbf{t}_{i}=\mathbf{K}_{i} \mathbf{w}_{i}$, with $\mathbf{K}_{i}$ the second order stiffness tensor for the $i^{\text {th }}$ pair, and assuming that the discrete-continuum maps (5), under the mentioned kinematical constraints, also hold for the actual kinematical descriptors, the constitutive relationships of the equivalent second-gradient continuum can be obtained in the form:

$$
\begin{aligned}
& \mathbf{T}=\mathrm{A} \mathbf{E}+\mathrm{B} \nabla^{2} \mathbf{w} \\
& \mathrm{T}=\mathrm{E} \mathbf{E}+\mathrm{F} \nabla^{2} \mathbf{w},
\end{aligned}
$$

where the elastic tensors of order four (A), five (B, E) and six (F) have components depending on the elastic constants of the matrix and on the geometry of the inclusions. For these tensors the same symbols as those used for the first order continuum are used in order to underline the similarities in the identification process, although their components in general differ. Their explicit expressions are reported in [33]. In the case of hyperelastic materials the transposition relation holds: $\mathbf{B A} \cdot \mathbf{B}=\mathbf{A} \cdot \mathbf{E} \mathbf{B}$, for any pair of second order tensors $\mathbf{A}$ and $\mathbf{B}$. In the case of central symmetry the odd order tensors B and E are null. These tensors and the tensor F contain material internal lengths. 
Then, the power density of the internal actions of the equivalent second gradient continuum can be written:

$$
\pi=\left[\mathrm{A} \mathbf{E}+\mathrm{B} \nabla^{2} \mathbf{w}\right] \cdot \mathbf{E}+\left[\mathrm{E} \mathbf{E}+\mathrm{F} \nabla^{2} \mathbf{w}\right] \cdot \nabla^{2} \mathbf{w} .
$$

It is useful to separate the different terms:

$$
\begin{aligned}
\pi^{Y Y} & =\mathrm{A} \mathbf{E} \cdot \mathbf{E} \\
\pi^{\nabla \nabla} & =\mathrm{F} \nabla^{2} \mathbf{w} \cdot \nabla^{2} \mathbf{w} \\
\pi^{\nabla Y} & =\mathrm{B} \nabla^{2} \mathbf{w} \cdot \mathbf{E}=\mathrm{E} \mathbf{E} \cdot \nabla^{2} \mathbf{w}=\pi^{Y \nabla},
\end{aligned}
$$

in such a way that:

$$
\pi=\pi^{Y Y}+\pi^{\nabla \nabla}+2 \pi^{Y \nabla}
$$

The term $\pi^{Y Y}$ corresponds to the classical term. The term $\pi^{\nabla \nabla}$ is characteristic to the second gradient continuum. The mixed term $\pi^{Y \nabla}$ is null in the case of centrosymmetric materials.

\subsection{Classical continum approximation}

Under both the constraints $\mathbf{d}=\mathbf{0}$ and $\mathbf{W}=\mathbf{R}$, assuming homogeneous discretecontinuum maps, that is Equations (5) with $\nabla^{2} \mathbf{w}=\mathbf{0}$, it is:

$$
\mathbf{w}_{i}=\mathbf{E}(\mathbf{a}-\mathbf{b}) .
$$

and the mean internal power (Equation 17) reads:

$$
\bar{\Pi}_{\mu}=\frac{1}{V\left(\mathscr{M}_{\mu}\right)} \sum_{i} \mathbf{t}_{i} \otimes(\mathbf{a}-\mathbf{b}) \cdot \mathbf{E}
$$

The kinematic map (24) corresponds to the so-called Cauchy-Born rule used in crystal elasticity.

Superimposing the continuous neighborhood of $\mathbf{x}, \mathscr{M}$, to the module, $\mathscr{M}_{\mu}$, the tensor $\mathbf{E}$ can be interpreted as the smooth field representing the symmetric strain measure of a Cauchy continuum. By requiring the equivalence between $\bar{\Pi}_{\mu}$ and the internal power density of the classical continuum

$$
\pi=\mathbf{T} \cdot \mathbf{E}
$$

for any $\mathbf{E}$, always basing on the localization theorem, the classical dual stress tensor $\mathbf{T}$ is identified as:

$$
\mathbf{T}=\frac{1}{V\left(\mathscr{M}_{\mu}\right)} \operatorname{sym}\left[\mathbf{t}_{i} \otimes(\mathbf{a}-\mathbf{b})\right]
$$


Assuming the linear elastic response functions for the contact actions as in Subsection 3.2 and assuming that the discrete-continuum maps (5), under the given kinematical constraints, also hold for the actual kinematical descriptors, the constitutive relationships of the equivalent classical continuum can be expressed in the form:

$$
\mathbf{T}=\mathrm{A} \mathbf{E},
$$

where $\mathrm{A}$ is the fourth order classical elastic tensor, which does not contain any material length, and:

$$
\pi=\pi^{Y Y}
$$

The Cauchy model is equivalent in terms of power to a discrete system of rigid particles locally constrained to have the same rotation which locally interact through forces.

\section{Structure of external power and balance equations for bulk and contact actions of the equivalent non-classical continua}

The structure of a non-local, scale-dependent, non-classical continuum is encoded in its internal power formula. In Section 3 it has been shown that the power equivalence between complex lattice systems and non-classical continua with additional degrees of freedom, together with the selection of response functions for the discrete model, leds to the identification of the constitutive functions for all the, standard and non-standard, internal actions. This is a key critical point for such kind of continua.

Other critical points are the derivation of the whole set of balance equations and the identification of the external actions $[5,15,6]$. In this Section it is shown how starting from the power density formula of continua as those identified in Section 3 , using the divergence theorem and applying the virtual power principle the structure of the corresponding external power can be defined, as well as the kinds of bulk and contact actions (macro and micro tractions). Moreover, in agreement with the axiomatic framework delineated in [13, 9], the local balance equations for the standard and non-standard actions are derived.

\subsection{Continuum with rigid and affine microstructure}

Let us now consider the internal power of the continuum identified in Subsection 3.1 over a control region $\mathscr{P} \subseteq \mathscr{C}, \mathscr{C}$ being the Euclidean region occupied by a body, with smooth boundary $\partial \mathscr{P}$ and outward normal $\mathbf{n}$ : 


$$
\Pi=\int_{\mathscr{P}}\left[\mathbf{S} \cdot(\nabla \mathbf{w}-\mathbf{W})+\frac{1}{2} S \cdot \nabla \mathbf{W}+\mathbf{z} \cdot \mathbf{d}+\mathbf{Z} \cdot \nabla \mathbf{d}\right] d V .
$$

The divergence theorem gives:

$$
\begin{aligned}
\Pi & =\int_{\mathscr{P}}\left[\operatorname{div} \mathbf{S} \cdot \mathbf{w}+\left(\frac{1}{2} \operatorname{div} \mathbf{S}+\operatorname{skw} \mathbf{S}\right) \cdot \mathbf{W}+(\operatorname{div} \mathbf{Z}-\mathbf{z}) \cdot \mathbf{d}\right] d V \\
& +\int_{\partial \mathscr{P}}\left(\mathbf{S} \mathbf{n} \cdot \mathbf{w}+\frac{1}{2} \mathrm{~S} \mathbf{n} \cdot \mathbf{W}+\mathbf{Z n} \cdot \mathbf{d}\right) d A
\end{aligned}
$$

Then the power equivalence between the internal and external power required for any $\mathbf{w}, \mathbf{W}$, and $\mathbf{d}$, provides the structure of the external power as:

$$
\Pi^{e}=\int_{\mathscr{P}} \mathbf{b} \cdot \mathbf{w} d V+\int_{\partial \mathscr{P}}\left(\mathbf{t} \cdot \mathbf{w}+\frac{1}{2} \mathbf{C} \cdot \mathbf{W}+\mathbf{p} \cdot \mathbf{d}\right) d A,
$$

for any $\mathscr{P} \subseteq \mathscr{C}$, where, for the sake of simplicity, neither volume terms dual to $\mathbf{W}$ nor volume terms dual to $\mathbf{d}$ (external volume microforces) are considered. By localization, the balance equations for the bulk:

$$
\begin{aligned}
& \operatorname{div} \mathbf{S}+\mathbf{b}=\mathbf{0}, \\
& \operatorname{div} S+2 \operatorname{skw} \mathbf{S}=\mathbf{0}, \quad \text { in } \mathscr{P} \\
& \operatorname{div} \mathbf{Z}-\mathbf{z}=\mathbf{0},
\end{aligned}
$$

and the contact actions (macrotractions, surface microcouples, microtractions):

$$
\mathbf{S n}=\mathbf{t}, \quad \mathbf{S n}=\mathbf{C}, \quad \mathbf{Z n}=\mathbf{p}, \quad \text { on } \partial \mathscr{P}
$$

are then derived. In Equations (33) and (34): $\mathbf{b}$ is the vector of the external volume forces; $\mathbf{t}$ and $\mathbf{C} \in \mathrm{Skw}$ are the vector and tensor (Skw being the set of second order skew-symmetric tensors) of surface forces and couples on $\partial P$, respectively; $\mathbf{p}$ is the vector of surface microforces exerted through $\partial \mathscr{P}$.

Equation (33a) expresses the classical linear momentum balance, (33b) the angular momentum balance and (33c) the micro linear momentum balance. It is worth noting that this last balance equation, obtained via the virtual power equivalence, is not obtainable via the standard invariance under Galilean changes of observers [15]. In Equations (33) and (34), $\mathbf{S}$ represents the second order macrostress tensor, $\mathbf{S}$ the third order couple-stress tensor, while $\mathbf{z}$ and $\mathbf{Z}$ are respectively the vector of the internal volume microstructural actions and the second order microstress tensor. These last terms account for the additional state of stress on the body due to the presence of defects and to their interactions. In particular, the internal force $\mathbf{z}$ can be interpreted as an auto-force accounting for the internal changes of the material configurations due to the presence of defects, while it can be shown that the stress tensor $\mathbf{Z}$, due to the relative deformation between defects, is related to the so-called configurational, or material, tensor [14, 21].

It can be shown that the microstrain measures $\mathbf{d}$ and $\nabla \mathbf{d}$ are non-null under a rigid micromotion. According to the axiomatic description in [9], it must be then 
required that the internal power under rigid macro and micro motions:

$$
\Pi^{*}=\int_{\mathscr{P}}\left[\mathbf{S} \cdot(\nabla \mathbf{w}-\mathbf{W})^{*}+\frac{1}{2} \mathrm{~S} \cdot \nabla \mathbf{W}^{*}+\mathbf{z} \cdot \mathbf{d}^{*}+\mathbf{Z} \cdot \nabla \mathbf{d}^{*}\right] d V
$$

is null for any strain field defined as in [31]: $(\nabla \mathbf{w}-\mathbf{W})^{*}=\mathbf{0}, \nabla \mathbf{W}^{*}=\mathbf{0}, \mathbf{d}^{*}=\mathbf{R d}$, $\nabla \mathbf{d}^{*}=\mathbf{R} \nabla \mathbf{d}$, where the symbol ' $*$ 'stands for the attribute 'rigid'. Then applying the divergence theorem it is:

$$
\begin{aligned}
\Pi^{*} & =\int_{\mathscr{P}}(\mathbf{z} \cdot \mathbf{R d} \cdot+\mathbf{Z} \cdot \mathbf{R} \nabla \mathbf{d}) d V \\
& =-\int_{\mathscr{P}} \mathbf{R z} \cdot \mathbf{d} d V+\int_{\mathscr{P}} \operatorname{div}(\mathbf{R Z}) \cdot \mathbf{d} d V-\int_{\mathscr{P}} \mathbf{R Z} \mathbf{n} \cdot \mathbf{d} d V \\
& =\int_{\mathscr{P}} \mathbf{R} \cdot(\operatorname{div} \mathbf{Z}-\mathbf{z}) \otimes \mathbf{d} d V+\int_{\partial P} \mathbf{R} \cdot \mathbf{Z n} \otimes \mathbf{d} d A=0,
\end{aligned}
$$

and, accounting for the microforce balance (Equation 33c), it is:

$$
\operatorname{skw}(\mathbf{Z n} \otimes \mathbf{d})=\mathbf{0}, \quad \text { on } \partial \mathscr{P} .
$$

Equation (37) is a micromoment balance equation, playing the role of a constitutive prescription [9], which implies that the microtraction $\mathbf{p}$ is parallel to $\mathbf{d}$.

If only the rigid microstructure is present $(\mathbf{d}=\mathbf{0})$, the internal power is zero for any rigid velocity field, and no equation must be added to Equations (33). In this case the bulk balance equations obtained using the principle of virtual power correspond to those of a micropolar continuum (33a,b), with the surface balance $(34 a, b)$.

\subsection{Second gradient continuum}

The internal power of a second-gradient continuum (Subsection 3.2) writes:

$$
\Pi=\int_{\mathscr{P}} \mathbf{T} \cdot \nabla \mathbf{w} d V+\int_{\mathscr{P}} \mathrm{T} \cdot \nabla^{2} \mathbf{w} d V
$$

where $\mathscr{P} \subseteq \mathscr{C}$ is the control volume with boundary $\partial P$ and outward normal $\mathbf{n}, \mathscr{C}$ always being the Euclidean region occupied by a body.

Applying the divergence theorem to the term related to the microtraction $\mathrm{T}$ it is:

$$
\int_{\mathscr{P}} \mathrm{T} \cdot \nabla^{2} \mathbf{w} d V=-\int_{\mathscr{P}} \operatorname{div} \boldsymbol{\top} \cdot \nabla \mathbf{w} d V+\int_{\partial \mathscr{P}} \mathrm{Tn} \cdot \nabla \mathbf{w} d A
$$

and, by putting $\tilde{\mathbf{T}}=\mathbf{T}-\operatorname{divT}$, Equation (38) can be rewritten as follows:

$$
\Pi=\int_{\mathscr{P}} \tilde{\mathbf{T}} \cdot \nabla \mathbf{w} d V+\int_{\partial \mathscr{P}} \mathrm{T} \mathbf{n} \cdot \nabla \mathbf{w} d A .
$$


Then, applying the divergence theorem to the first term of Equation (40), it is:

$$
\int_{\mathscr{P}} \tilde{\mathbf{T}} \cdot \nabla \mathbf{w} d V=-\int_{\mathscr{P}} \operatorname{div} \tilde{\mathbf{T}} \cdot \mathbf{w} d V+\int_{\partial \mathscr{P}} \tilde{\mathbf{T}} \mathbf{n} \cdot \mathbf{w} d A .
$$

By decomposing $\nabla \mathbf{w}$ as: $\nabla \mathbf{w}=\nabla_{s} \mathbf{w}+\partial_{n} \mathbf{w} \otimes \mathbf{n}$, with $\nabla_{s} \mathbf{w}=\nabla \mathbf{w}(\mathbf{I}-\mathbf{n} \otimes \mathbf{n})$ and $\partial_{n} \mathbf{w}=\nabla \mathbf{w} \mathbf{n}, \mathbf{I}$ being the identity tensor, the expression of the internal power (40) becomes:

$\Pi=-\int_{\mathscr{P}} \operatorname{div} \tilde{\mathbf{T}} \cdot \mathbf{w} d V+\int_{\partial \mathscr{P}} \tilde{\mathbf{T}} \mathbf{n} \cdot \mathbf{w} d A+\int_{\partial \mathscr{P}} \operatorname{Tn} \cdot \nabla_{s} \mathbf{w} d A+\int_{\partial \mathscr{P}}(\mathbf{T} \mathbf{n}) \mathbf{n} \cdot \partial_{\mathbf{n}} \mathbf{w} d A$.

The theorem of divergence can also be applied to the term of Equation (42) related to the surface velocity gradient in several ways [23, 12, 28]. One way consists in exploiting the surface divergence theorem [12]:

$$
\int_{\partial_{\mathscr{P}}} \operatorname{Tn} \cdot \nabla_{S} \mathbf{w} d A=-\int_{\partial \mathscr{P}}\left[\operatorname{div}_{S}(\operatorname{Tn})+2 k(\operatorname{Tn}) \mathbf{n}\right] \cdot \mathbf{w} d A,
$$

and then:

$\Pi=-\int_{\partial \mathscr{P}} \operatorname{div} \tilde{\mathbf{T}} \cdot \mathbf{w}+\int_{\partial \mathscr{P}}\left[\tilde{\mathbf{T}} \mathbf{n}-\operatorname{div}_{S}(\operatorname{Tn})-2 k(\mathbf{T n}) \mathbf{n}\right] \cdot \mathbf{w} d A+\int_{\partial \mathscr{P}}(\operatorname{Tn}) \mathbf{n} \cdot \partial_{\mathbf{n}} \mathbf{w} d A$,

where $k=-\frac{1}{2} \operatorname{tr} \nabla_{s} \mathbf{n}$ is the mean curvature of $\partial \mathscr{P}$. In this case the power equivalence between internal and external power required for any $\mathbf{w}, \mathbf{W}$ and $\mathbf{d}$, and any $\mathscr{P} \subseteq \mathscr{C}$, provides the structure of the external power as:

$$
\Pi^{e}=\int_{\mathscr{P}} \mathbf{b} \cdot \mathbf{w} d V+\int_{\partial \mathscr{P}}\left(\mathbf{f} \cdot \mathbf{w}+\mathbf{h} \cdot \partial_{\mathbf{n}} \mathbf{w}\right) d A,
$$

where $\mathbf{b}$ is the body force, $\mathbf{f}$ and $\mathbf{h}$ are the diffused traction and microtractions on d $\mathscr{P}$, respectively.

By localisation the balance equations for the bulk and contact actions become:

$$
\operatorname{div} \tilde{\mathbf{T}}+\mathbf{b}=\mathbf{0} \quad \text { in } \mathscr{P},
$$

with:

$$
\begin{aligned}
\tilde{\mathbf{T}} \mathbf{n}-\operatorname{div}_{s} \operatorname{Tn}-2 k(\operatorname{Tn}) \mathbf{n} & =\mathbf{f}, \\
(\operatorname{Tn}) \mathbf{n} & =\mathbf{h} \quad \text { on } \quad \partial \mathscr{P} .
\end{aligned}
$$

Another way of applying the divergence theorem [28] accounts for the presence of contact actions distributed along lines $\partial(\partial \mathscr{P})$ in such a way that:

$$
\int_{\partial_{\mathscr{P}}} \operatorname{Tn} \cdot \nabla_{S} \mathbf{w} d A=-\int_{\partial \mathscr{P}} \operatorname{div}_{S}(\operatorname{Tn}) \cdot \mathbf{w} d A+\int_{\partial(\partial \mathscr{P})}(\operatorname{Tn}) \mathbf{m} \cdot \mathbf{w} d l
$$


where $\mathbf{m}$ is a unit vector, orthogonal to both $\mathbf{n}$ and the tangent direction of $\partial(\partial P)$, pointing outward from the interior of $\partial P$. Considering and edge $\widehat{\partial \mathscr{P}}$ with outwards normals $\mathbf{n}$ and $\mathbf{m}$ are not univocally defined it is assumed that:

$$
\int_{\partial(\partial \mathscr{P})}(T \mathbf{n}) \mathbf{m} \cdot \mathbf{w} d l=\int_{\widehat{\partial \mathscr{P}}}<(T \mathbf{n}) \mathbf{m}>\cdot \mathbf{w} d l,
$$

where $<(T \mathbf{n}) \mathbf{m}>$ denotes the edge average of $(T \mathbf{n}) \mathbf{m}$ over the tangent and the normal vectors of the two surfaces connecting at $\widehat{\partial \mathscr{P}}$. Then the internal power can be rewritten as:

$$
\begin{aligned}
\Pi & =-\int_{\mathscr{P}} \operatorname{div} \tilde{\mathbf{T}} \cdot \mathbf{w} d V+\int_{\partial \mathscr{P}}\left[\tilde{\mathbf{T}} \mathbf{n}-\operatorname{div}_{S}(\operatorname{Tn})\right] \cdot \mathbf{w} d A \\
& +\int_{\partial \mathscr{P}}(\operatorname{Tn}) \mathbf{n} \cdot \partial_{\mathbf{n}} \mathbf{w} d A+\int_{\widehat{\partial \mathscr{P}}}<(\mathbf{T} \mathbf{n}) \mathbf{m}>d l
\end{aligned}
$$

Applying the virtual power principle the external power can be written as:

$$
\Pi^{e x t}=\int_{\mathscr{P}} \mathbf{b} \cdot \mathbf{w} d V+\int_{\partial \mathscr{P}}\left(\mathbf{f} \cdot \mathbf{w}+\mathbf{h} \cdot \partial_{\mathbf{n}} \mathbf{w}\right) d A+\int_{\widehat{\partial \mathscr{P}}} \mathbf{h}_{l} \cdot \mathbf{w} d l .
$$

where $\mathbf{b}$ is the body force, $\mathbf{f}$ and $\mathbf{h}$ are the diffused traction and microtraction on $\partial \mathscr{P}$, respectively, and $\mathbf{h}_{l}$ is the traction concentrated at the edge $\widehat{\partial \mathscr{P}}$. By localising, the local balance equations derived are:

$$
\operatorname{div} \tilde{\mathbf{T}}+\mathbf{b}=\mathbf{0} \quad \text { in } \mathscr{P},
$$

with:

$$
\begin{aligned}
\tilde{\mathbf{T}} \mathbf{n}-\operatorname{div}_{s}(\mathbf{T} \mathbf{n}) & =\mathbf{f}, \\
(\mathbf{T}) \mathbf{n} & =\mathbf{h} \quad \text { on } \quad \partial \mathscr{P}, \\
<(T \mathbf{n}) \mathbf{m}> & =\mathbf{h}_{l} \quad \text { on } \widehat{\partial \mathscr{P} .}
\end{aligned}
$$

In the case of the internal power of the continuum of Subsection 3.3, the virtual power principle gives the balance equations of the Cauchy continuum:

$$
\begin{array}{rlll}
\operatorname{div} \mathbf{T}+\mathbf{b}=\mathbf{0} & \text { in } \quad \mathscr{P} . \\
\mathbf{T n}=\mathbf{f} & \text { on } & \partial \mathscr{P} .
\end{array}
$$




\section{Numerical simulations}

In this paragraph some results of numerical simulations, obtained using COMSOL Multiphysics(c) finite element code, are mentioned for highlighting the potentiality of the non-classical continua described in Section 3 and 4.

\subsection{Porous fibre reinforced composites}

The main features of the non-classical continua described above, here also called multifield continua because of the presence of additional degrees of freedom, are the presence of internal lengths in the material description and spatial dispersion in wave propagation. For these reasons such continua can be classified as 'implicitly' non-local models [18, 10].

In the continuum with rigid and affine microstructure of Sections 3.1 and 4.1 dispersion properties are related to the presence of the microvelocity term $\mathbf{d}$ in the equations of motion, that is not a derivative nor in space neither in time. In the works $[34,31]$ a one-dimensional problem, a bar with continuous distribution of microcraks, has been analysed under the effect of free and forced oscillations. In both cases, the variation of the phase velocity of propagating waves with the frequency, or the wave number, showed that the additional descriptor $\mathbf{d}$ reveals the presence of the microcrack as a disturbance spread along the bar which alters the shape and the velocity of the waves, and the that the kind of this disturbance strongly depends on the microcrack density per unit lengths.

Here the results of a two dimensional panel made of an orthotetragonal porous ceramic material in tension has been reported in order to show another peculiarity of the multifield continuum of Sections 3.1 and 4.1 that is the reduction in stiffness obtained as an effect of the additional stress/strain state introduced in the multifield model.

The panel, of length $L=100 \mu \mathrm{m}$, is simply supported and has different levels of porosity, evaluated with a pore density factor $p$ (pores area/panel area). In the multifield model this factor enters into the constitutive tensors $\mathrm{M}$ and $\mathrm{R}$ of Equations (11). It is made of $\mathrm{Al}_{2} \mathrm{O}_{3}$ hexagonal grains, of side $22.5 \mu \mathrm{m}$, and $\mathrm{Co}$ interfaces, of thickness $1-2 \mu \mathrm{m}$, with Youngs' modulus and Poisson's coefficients: $E_{g}=410000 \mathrm{MPa}$, $v_{g}=0.25$ and $E_{i}=210000 \mathrm{MPa}, v_{i}=0.235$, respectively. The grains are not rigid and their deformability has be taken into account in terms of energy equivalent stiffness at the interfaces: $A_{i} E_{i} E_{g} /\left(E_{i} A_{i}+E_{g} A_{g}\right), A_{g}$ and $A_{i}$ being the grain and interface areas, respectively. The multifield solution has been obtained and compared with a Finite Element solution for: $p=0.039(1), p=0.11(2), p=0.192$ (3), $p=0.254$ (1). The two solutions show that the vertical component of the displacement increases with the increase of the porosity (Figure 2), confirming that in such a multifield model the presence of damage can be accounted for as an additional state of stress and strain rather than a reduction in stiffness, like in internal variables models with which these results have been compared [17]. 

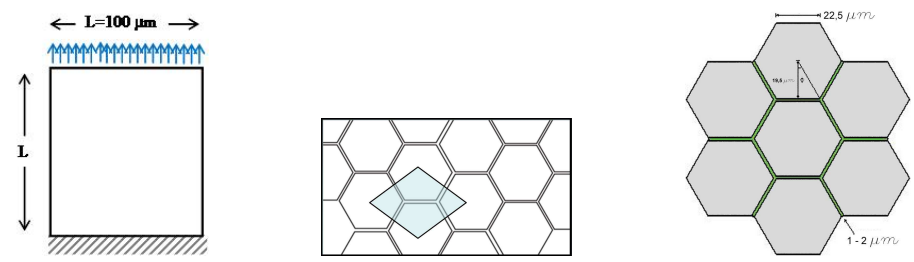

Fig. 1 Sketch of the ceramic panel
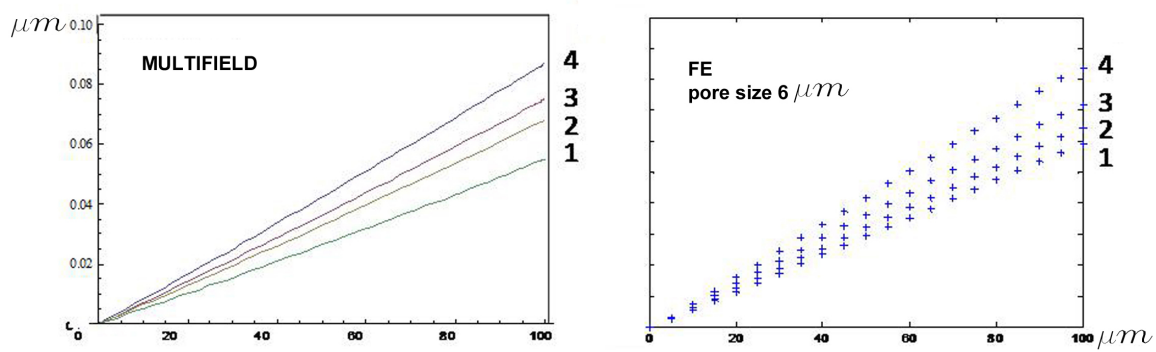

Fig. 2 Vertical component of displacement along the vertical direction of the panel for different pore density: (1) $p=0.039$, (2) $p=0.11$, (3) $p=0.192$, (4) $p=0.254$

\subsection{Masonry-like materials}

Here some results of a parametric study conducted for various schemes of orthotropic block assemblies is reported. A square panel of side $L$, made of blocks of length $b$ and height $h$, simply supported and subjected to shear load has been analysed by varying the scale $\left(\varepsilon_{1}=b / L, \varepsilon_{2}=h / L\right)$ and aspect $(\rho=h / b)$ ratios (Figure 3). The panel has been described as a discrete model made of rigid bodies interacting by linear elastic springs and as a Cosserat, a second gradient and a Cauchy model, as identified in Sections 3, 4.

Figure 4 shows the contours lines of the angular component of the strain tensors, in the discrete, Cosserat, $\left([\nabla \mathbf{w}-\mathbf{W}]_{12}=\nabla \mathbf{w}-\mathbf{W} \cdot \mathbf{e}_{1} \otimes \mathbf{e}_{2}\right)$, second gradient and Cauchy $\left([\mathbf{E}]_{12}=\mathbf{E} \cdot \mathbf{e}_{1} \otimes \mathbf{e}_{2}\right), \mathbf{e}_{1}, \mathbf{e}_{2}$ being the unit vectors defining the horizontal and vertical direction, respectively, obtained for the cases: $a 1\left(\varepsilon_{1}=0.2, \varepsilon_{2}=0.05\right.$, $\rho=0.25) ; a 4\left(\varepsilon_{1}=0.2, \varepsilon_{2}=0.1, \rho=0.5\right) ; b 7\left(\varepsilon_{1}=0.2, \varepsilon_{2}=0.2, \rho=1\right)$. It can be noted that the Cosserat continuum solution is always in good agreement with the discrete solution. Differently, the second gradient and Cauchy continua fit well the response of the discrete model only in the orthotetragonal case (square blocks with no-interlocking: case $b 7$ ).

The cases of Figure 5: $a 3\left(\varepsilon_{1}=0.025, \varepsilon_{2}=0.0125, \rho=0.25\right) ; a 6\left(\varepsilon_{1}=0.025\right.$, $\left.\varepsilon_{2}=0.025, \rho=0.5\right) ; b 9\left(\varepsilon_{1}=0.025, \varepsilon_{2}=0.025, \rho=1\right)$ show that the same occurs at finer scales: the differences between the Cauchy/second gradient and dis- 

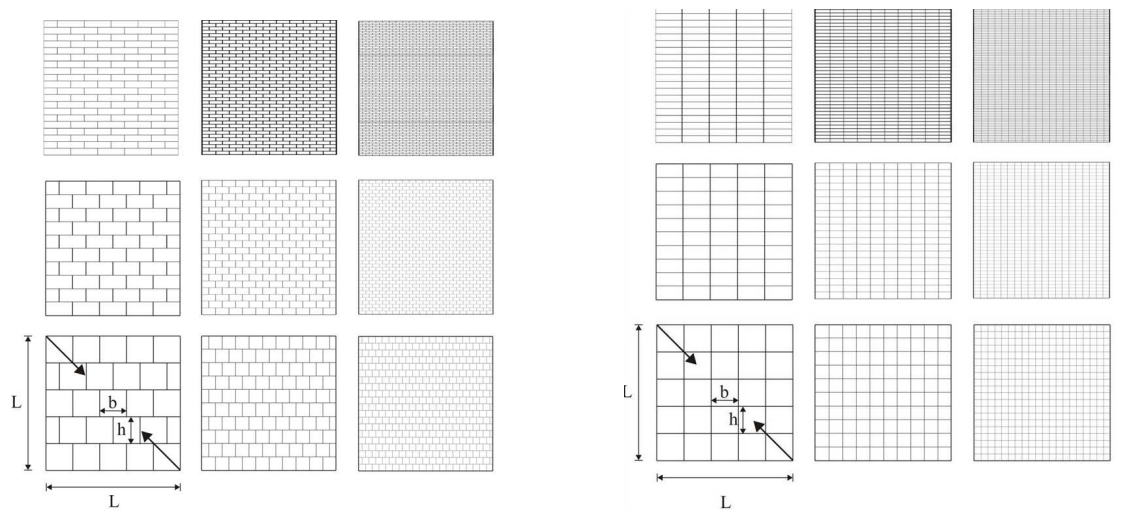

Fig. 3 Orthotropic block assemblies with different scale and aspect ratios. Left, systems with interlocking (from top to bottom and left to right: schemes a1-a9). Right, systems without interlocking (from top to bottom and left to right: schemes b1-b9)
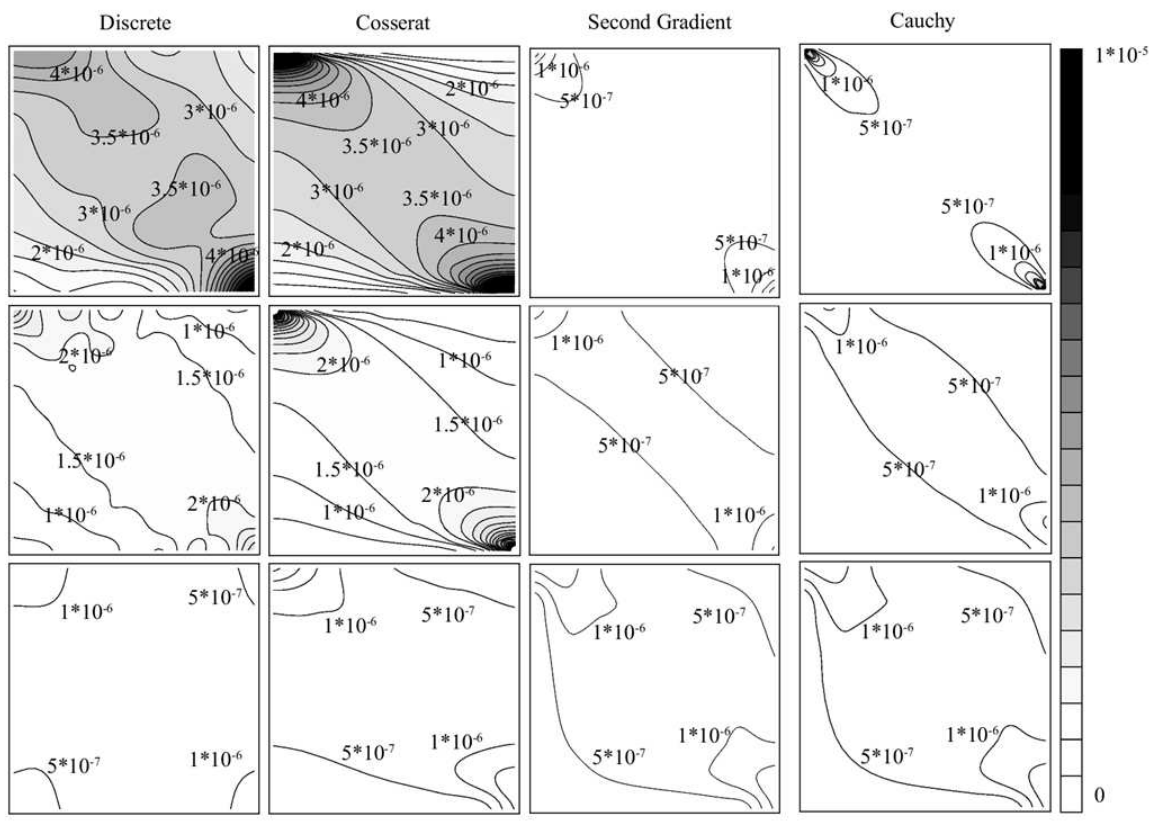

Fig. 4 Contour lines of the angular strain component in the discrete, Cosserat $\left([\nabla \mathbf{w}-\mathbf{W}]_{12}\right)$, second gradient and Cauchy ([E $\left.]_{12}\right)$ model. Cases: $a 1, a 4, b 7$ [33] 


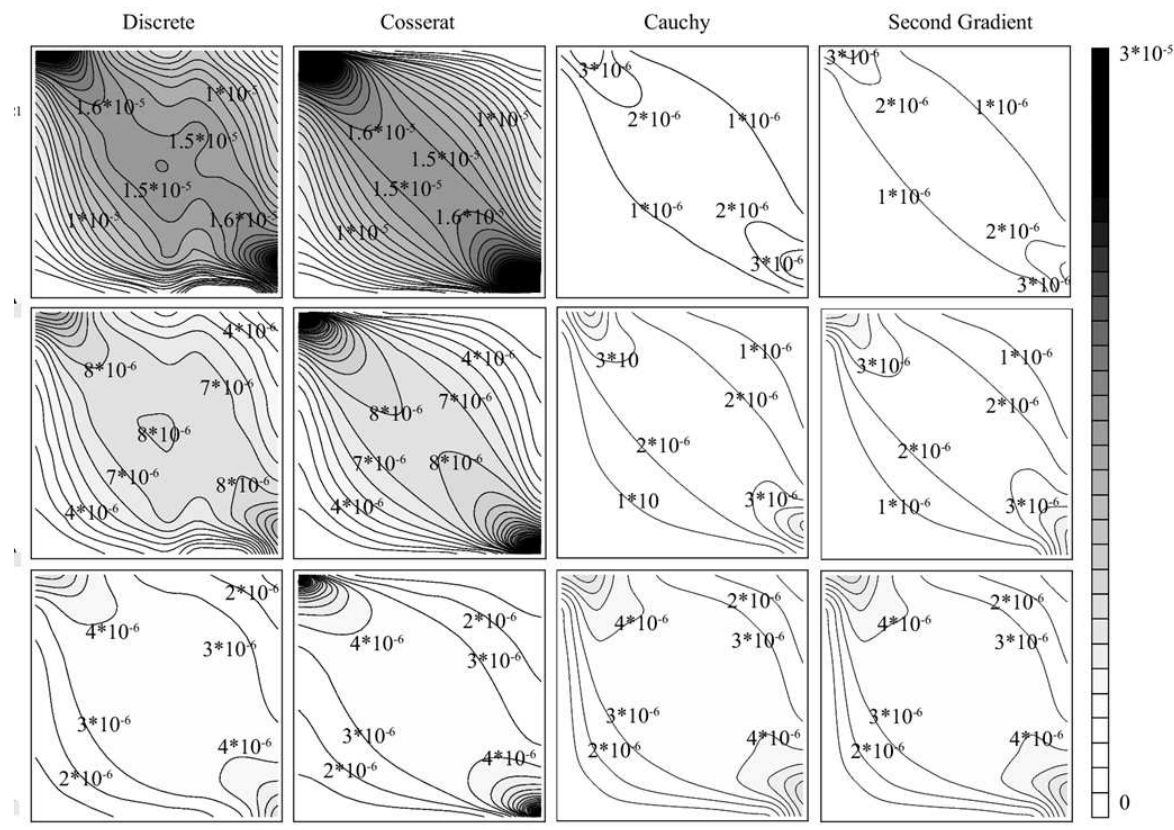

Fig. 5 Contour lines of the angular strain component in the discrete, Cosserat $\left([\nabla \mathbf{w}-\mathbf{W}]_{12}\right)$, second gradient and Cauchy ([E $\left.]_{12}\right)$ model. Cases: $a 3, a 6, b 9$ [33]

crete/Cosserat solutions are reduced, but still remain. The correspondence of all the solutions is obtained only in the orthotetragonal case.

The reason for which second gradient and Cauchy models fail in representing the behaviour of the discrete systems for orthotropic materials relies in the strong nonsymmetries of the strain and stress tensors. In the discrete as in the Cosserat models in fact the power term $\pi^{K K}$ due to relative rotation, which corresponds to the skewsymmetric part of the strain, plays an important role. Note that in the orthotetragonal case it is always $\pi^{Y K}=\pi^{K Y}=0$ (see Equations 14). In the second gradient and Cauchy continua instead the strain, as well as the stress, is symmetric and the relative rotation term is not present (Equations 22). This can be also confimed by the results of Figure 6 showing the contour lines of the sole non-null component of the relative rotation in the Cosserat model $\left([\mathbf{R}-\mathbf{W}]_{12}=(\mathbf{R}-\mathbf{W}) \cdot \mathbf{e}_{1} \otimes \mathbf{e}_{2}=-[\mathbf{R}-\mathbf{W}]_{12}=(\mathbf{R}-\right.$ $\left.\mathbf{W}) \cdot \mathbf{e}_{2} \otimes \mathbf{e}_{1}\right)$. Here the cases shown are: $a 1\left(\varepsilon_{1}=0.2, \varepsilon_{2}=0.05, \rho=0.25\right) ; a 7$ $\left(\varepsilon_{1}=0.2, \varepsilon_{2}=0.2, \rho=1\right) ; b 7\left(\varepsilon_{1}=0.2, \varepsilon_{2}=0.2, \rho=1\right) ; a 3\left(\varepsilon_{1}=0.025, \varepsilon_{2}=\right.$ $0.0125, \rho=0.25) ; a 9\left(\varepsilon_{1}=0.025, \varepsilon_{2}=0.025, \rho=1\right) ; b 9\left(\varepsilon_{1}=0.025, \varepsilon_{2}=0.025\right.$, $\rho=1)$. This component reduces with the loss of interlocking becoming null in the orthotetragonal case $(b 7, b 9)$.

Overall, this parametric study shows that the Cosserat continuum works well both in case of particles of significant size and when it is necessary to account for strong non-symmetries in strain and stress, like in orthotetragonal assemblies. The second 


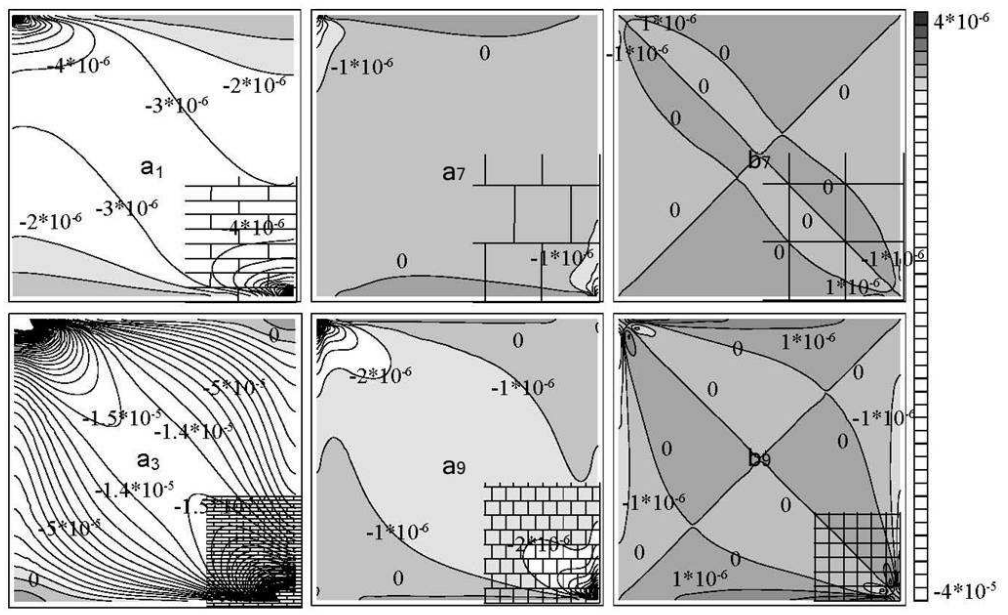

Fig. 6 Contour lines of the relative rotation in the Cosserat model. Cases: $a 1, a 7, b 7, a 3, a 9, b 9$ (courtesy of A. Pau)

gradient continuum, differently from the classical continuum, can represent the scale effects but, as the Cauchy continuum, lacks of the descriptor for the relative rotation (i.e. strain non-symmetry) that can be predominant in orthotropic assemblies.

\section{Final remarks}

The discrete modelling of materials, crucial in the past for building constitutive theories for solids, can still be of help in determining physically plausible constitutive models for complex materials. The most significant suggestion, in a sense derived by Voigt and Poincaré, is the idea to build-up a refined non-local intermolecular potential based on appropriate, physically based, complex discrete systems to define case by case. Where refined here means to extend the concept of 'molecule' for representing the various internal phases and to use generalised correspondence maps between discrete and continuum descriptors.

Scale-dependent continuous macro models have been unambiguously identified from complex discrete micro-models using the power equivalence procedure described in Section 3 using generalised correspondence laws between the large set of degrees of freedom of the discrete and the continuum field descriptors. In this way the macroscopic stress measures have been identified in terms of the constitutive constants and the geometry of the micro-model. In order to provide physical consistency to multifield continua, the constitutive relations have been finally derived assuming physically-based response functions for the lattice interactions. 
These continua retains memory of the fine organization of the material by means of additional field descriptors and satisfy the basic requirements for the mechanical modelling of complex materials, that is: the presence of internal lengths and spatial dispersion in wave propagation, which in turn define the non-local character of the material description.

Acknowledgements This research has been partially supported by the Italian 'Ministero dell'Università e della Ricerca Scientifica' (Research fund: MIUR Prin 2010-11(/12)).

\section{References}

1. H. Altenbach and V. A. Eremeyev, editors. Generalized Continua from the Theory to Engineering Application, volume 541 of CISM Courses and Lectures. Springer, Berlin, 2013.

2. D. Capecchi, G. Ruta, and P. Trovalusci. From classical to Voigt's molecular models in elasticity. Archive for History of Exact Sciences, 64:525-559, 2010.

3. D. Capecchi, G. Ruta, and P. Trovalusci. Voigt and Poincaré's mechanistic-energetic approaches to linear elasticity and suggestions for multiscale modelling. Archive of Applied Mechanics, 81(11):1573-1584, 2011.

4. G. Capriz. Continua with latent microstructure. Archive for Rational Mechanics Analysis, 90:43-56, 1985.

5. G. Capriz. Continua with Microstructure. Springer-Verlag, Berlin, 1989.

6. G. Capriz and P. Podio-Guidugli. Whence the boundary conditions in modern continuum physics. In Atti dei Convegni Lincei, volume 210, pages 19-42, Roma, 2004. Accademia Nazionale dei Lincei.

7. A.-L. Cauchy. Sur l'équilibre et le mouvement d'un système de points matériels sollicités par des forces d'attraction ou de répulsion mutuelle. Exercices de Mathématiques, 3, 1822, 1827:188-213, 1828. Euvres 2 (8): 227-252.

8. S. Cowin and J. W. Nunziato. Linear elastic materials with voids. Journal of Elasticity, 13:15-147, 1983.

9. A. Di Carlo. A non-standard format for continuum mechanics. In R. C. Batra and M. F. Beatty, editors, Contemporary Research in the Mechanics and Mathematics of Materials, pages 92104, Barcelona (Spain), 1996. International Center for Numerical Methods in Engineering (CIMNE).

10. A. C. Eringen. Microcontinuum Field Theories. Springer-Verlag, New York, 1999.

11. M. Frémond and B. Nedjar. Damage, gradient of damage and principle of virtual power. International Journal of Solids and Structures, 33(8):1083-1103, 1996.

12. E. Fried and M. E. Gurtin. Tractions, balances and boundary conditions for non simple materials with application to liquid flow at small-length scales. Archive of Rational Mechanics, 182:513-554, 2006.

13. P. Germain. The method of virtual power in continuum mechanics. Part 2: Microstructure. SIAM Journal of Applied Mathematics, 25(3):556-575, 1973.

14. M. E. Gurtin. Configurational Forces as Basis Concept of Continuum Physics. SpringerVerlag, Berlin, 2000.

15. M. E. Gurtin and P. Podio-Guidugli. On the formulation of mechanical balance laws for structured continua. Zeitschrift Für Angewandte Mathematik und Physik, 43:181-190, 1992.

16. M. E. Gurtin and P. Podio-Guidugli. Configurational Forces and the Basic Laws for Crack Propagation. Journal of the Mechanics and Phisics of Solids, 44:905-927, 1996.

17. M. Kachanov. On the effective moduli of solids with cavities and cracks. International Journal of Fracture, 9:R17-R21, 1993. 
18. I.A. Kunin. Elastic Media with Microstructure-I, One-dimensional Models. Springer-Verlag, Berlin, 1982. (Russian edition 1975).

19. A. Mattoni, L. Colombo, and F. Cleri. Atomistic study of the interaction between a microcrack and a hard inclusion. Physical Review B, 70(9):094108, 2004.

20. G. A. Maugin. Nonlocal theories or gradient-type theories: a matter of convenience? Archives of Mechanics, 31(1):15-26, 1979.

21. G. A. Maugin. Configurational Forces. Thermomechanics, Physics, Mathematics, and Numerics. CRC Series: Modern Mechanics and Mathematics. Chapman and Hall, Boca Raton (FL), 2011.

22. R. D. Mindlin. Micro-structure in linear elasticity. Archive of Rational Mechanics and Analysis, 16(51-78), 1964.

23. R. D. Mindlin. Second gradient of strain and surface-tension in linear elasticity. International Journal of Solids and Structures, 1:417-438, 1965.

24. R. D. Mindlin and N. N. Eshel. On first strain-gradient theories in linear elasticity. International Journal of Solids and Structures, 4:109-124, 1968.

25. C.-L.-M.-H. Navier. Mémoire sur le lois de l'équilibre et du mouvement des corps solides élastiques (1821). In Mémoires de l'Academie des Sciences de l'Institut de France, volume 7 of $I I$, pages 375-393, 1827.

26. I. Newton. Opticks or a Treatise of the Reflections, Refractions, Inflections and Colours of Ligth. Queries, XXXI, $4^{\text {th }}$ ed. W. Innis (Royal Society), London, $1730(1717,1704)$.

27. J. W. Nunziato and S. Cowin. A nonlinear theory of elastic materials with voids. Archive for Rational Mechanics and Analysis, 72(2):175-201, 1979.

28. P. Podio-Guidugli and M. Vianello. Hypertractions and hyperstresses convey the same mechanical information. Continuum Mechanics and Thermodynamics, 22(3):163-176, 2010.

29. H. Poincaré. Leçons sur la Théorie de l'Élasticité. Georges Carré, Paris, 1892.

30. S.-D. Poisson. Mémoire sur l'équilibre et le mouvement des corps élastiques. In Mémoires de l'Académie des Sciences de l'Institut de France, volume 8, pages 357-405, 1829. Lu à l'Académie en 1828.

31. P. Trovalusci. Molecular approaches for multifield continua: origins and current developments. In T. Sadowsky and P. Trovalusci, editors, Multiscale Modeling of Complex Materials: phenomenological, theoretical and computational aspects, number 556 in CISM Courses and Lectures, pages 211-278. Springer, Berlin, 2014.

32. P. Trovalusci, D. Capecchi, and G. Ruta. Genesis of the multiscale approach for materials with microstructure. Archive of Applied Mechanics, 79:981-997, 2009.

33. P. Trovalusci and A. Pau. Derivation of microstructured continua from lattice systems via principle of virtual works. The case of masonry-like materials as micropolar, second gradient and classical continua. Acta Mechanica, 225(1):157-177, 2014.

34. P. Trovalusci, V. Varano, and G. Rega. A generalized continuum formulation for composite materials and wave propagation in a microcracked bar. Journal of Applied Mechanics, 77(6):061002-1/11,2010

35. W. Voigt. Theoretische Studien über die Elasticitätsverhältnisse der Kristalle. In Abhandlungen der Gesellschaft der Wissenschaften zu Göttingen, XXXIV, 1887.

36. W. Voigt. Lehrbuch der Kristallphysik. B. G. Teubner, Leipzig, 1910. 